\title{
Influence of Leg Muscle Vibration on Human Walking
}

\author{
Y. P. IVANENKO, R. GRASSO, AND F. LACQUANITI \\ Human Physiology Section of the Scientific Institute Santa Lucia and the University of Rome "Tor Vergata," \\ 00179 Rome, Italy
}

Received 8 March 2000; accepted in final form 1 June 2000

Ivanenko, Y. P., R. Grasso, and F. Lacquaniti. Influence of leg muscle vibration on human walking. J Neurophysiol 84: 1737-1747, 2000. We studied the effect of vibratory stimulation of different leg muscles [bilateral quadriceps (Q), hamstring (HS) muscles, triceps surae (TS), and tibialis anterior (TA)] in seven normal subjects during 1) quiet standing, 2) stepping in place movements, and 3) walking on the treadmill. The experiments were performed in a dimly illuminated room, and the subjects were given the instruction not to resist the applied perturbation. In one condition the velocity of the treadmill was controlled by a feedback from the subject's current position. In normal standing, TA vibration elicited a prominent forward body tilt, whereas HS and TS vibration elicited backward trunk or whole body inclination, respectively. Q vibration had little effect. During stepping in place, continuous HS vibration produced an involuntary forward stepping at about $0.3 \mathrm{~m} \mathrm{~s}^{-1}$ without modifying the stepping frequency. When the subjects (with eyes closed) kept a hand contact with an external still object, they did not move forward but perceived an illusory forward leg flexion relative to the trunk. Q, TS, and TA vibration did not cause any systematic body translation nor illusory changes in body configuration. In treadmill locomotion, HS vibration produced an involuntary steplike increase of walking speed (by $0.1-$ $\left.0.6 \mathrm{~m} \cdot \mathrm{s}^{-1}\right)$. Continuous vibration elicited larger speed increments than phasic stimulation during swing or stance phase. For phasic stimulation, HS vibration tended to be more effective when applied during swing than during stance phase. Q, TA, and TS vibration had little if any effect. Vibration of thigh muscles altered the walking speed depending on the direction of progression. During backward locomotion, the walking speed tended to decrease after HS vibration, whereas it significantly increased after Q vibration. Thus the influence of leg muscle vibration on stepping in place and locomotion differed significantly from that on normal posture. We suggest that the proprioceptive input from thigh muscles may convey information about the velocity of the foot movement relative to the trunk.

\section{N T R O D U C T I O N}

Although the central networks of coupled oscillators designated as central pattern generators (Grillner 1981) can autonomously generate patterns of rhythmic activity for locomotion, they are normally modulated by supraspinal inputs and sensory signals (Armstrong 1988; Grillner 1981; Lacquaniti et al. 1999; Orlovski et al. 1999; Pearson et al. 1998; Rossignol 1996; Shik and Orlovsky 1976). Proprioception plays a specific role in entraining the locomotor rhythm. During fictive or real locomotion, the periodic stretch or vibration of proximal and distal muscles can entrain or reset the locomotor rhythm, modulating the amplitude and phase of extensor and flexor activity (Con-

\footnotetext{
Address for reprint requests: Y. P. Ivanenko, Human Physiology Section, Scientific Institute Santa Lucia, via Ardeatina 306, 00179 Rome, Italy (E-mail: y.ivanenko@hsantalucia.it).
}

way et al. 1987; Hiebert et al. 1996). The passive extension of the hindlimbs involved in lifting a quiescent spinal cat triggers air-stepping; conversely, lowering an air-stepping cat to the ground and flexing the limbs slows or stops the rhythm. In the same animal preparation, manually flexing one hip during walking on the treadmill abolishes stepping in that limb, while stepping is resumed when the hip is extended steadily to the limit that is normally reached at the end of stance (Grillner and Rossignol 1978). Also, the hindlimbs of a spinal cat walking on a split-belt treadmill adapt their cadence to each belt separately (Forssberg et al. 1980). Therefore sensory signals from the moving limbs have access to the central locomotor networks to modulate walking speed, although the overall role of peripheral feedback in the regulation of the walking pattern may be very dependent on the type of preparation used (i.e., fictive locomotion or decerebrate walking or intact walking) (see Hiebert et al. 1996). In man, the continuous vibration of several lower limb muscles can produce involuntary rhythmic air-stepping of a suspended leg in horizontally lying humans (Gurfinkel et al. 1998).

Proprioceptive inputs are normally integrated with cutaneous, visual, and vestibular signals giving rise to a coherent body scheme for posture (including equilibrium maintenance) and movement (Gandevia 1996; Gurfinkel 1994; Lacquaniti 1997; Massion 1992; Soechting and Flanders 1992; Tiemersm 1989). One approach to probe the role of proprioception for posture and movement consists of applying vibrations to muscles or tendons (thereby activating mainly muscle spindle afferents) (Bianconi and van der Meulen 1963; Burke et al. 1976; Goodwin et al. 1972; Matthews and Stein 1969). Such stimulation has revealed some important properties of the motor control system and of the use of proprioceptive information. During simple voluntary arm movements, vibration of the lengthening antagonist muscle (and not the muscle acting as a prime mover) has been shown to be critical for accurate perception of movement and limb position (Bullen and Brunt 1986; Capaday and Cooke 1981; Cordo et al. 1995; Inglis et al. 1991). When applied to a standing human subject, muscle vibration induces several effects (from illusions of ego- or exo-motion to actual body tilt) that depend on the vibrated muscle, the sensory context and the task (Eklund 1972; Ivanenko et al. 1999a,c; Kavounoudias et al. 1999; Lackner and Levine 1979; Lekhel et al. 1997; Quoniam et al. 1990; Roll et al. 1989b; Smetanin et al. 1993). For example, shank muscle

\footnotetext{
The costs of publication of this article were defrayed in part by the payment of page charges. The article must therefore be hereby marked "advertisement" in accordance with 18 U.S.C. Section 1734 solely to indicate this fact.
} 
vibration evokes a prominent body tilt during quiet standing (Eklund 1972) or an illusion of body inclination in the opposite direction when the trunk is fixed (Lackner and Levine 1979). The influence of shank muscle vibration strikingly diminishes (Ivanenko et al. 1999c) when applied to a subject standing on an unstable movable support.

While stimulation of supraspinal centers has been shown to have profound influences both on posture and locomotion (see Mori et al. 1982), the influence of proprioceptive inputs evoked by muscle vibration on human locomotion has been studied to a much lesser extent (see, however, Gurfinkel et al. 1998; Ivanenko et al. 2000). Here we report the effects of applying mechanical vibrations to thigh and shank muscles in healthy humans who performed the following tasks: quiet standing, stepping in place, and walking on a treadmill at different speeds. The results demonstrated that the most prominent effect on human locomotion was observed during hamstring muscle vibration. We argue that the effect may be related to the mechanisms whereby body movement relative to the ground is represented. An abstract of these results has been published elsewhere (Ivanenko et al. 1999b).

\section{MET H O D S}

\section{Experimental setup}

The experiments were carried out both overground and on a treadmill (Woodway XELG 70, Germany). Horizontal body displacement and orientation of body segments relative to the vertical in the sagittal plane were monitored by means of the ELITE system (BTS, Milan, Italy). Kinematic data were digitized at $100 \mathrm{~Hz}$ and filtered with an optimal low-pass FIR filter with automatic bandwidth selection. General procedures have been previously described (Bianchi et al. 1998; Borghese et al. 1996). Four 100-Hz TV cameras were spaced on the recording side of the treadmill to enhance spatial accuracy. After three-dimensional calibration, the spatial accuracy of the system was better than $1.5 \mathrm{~mm}$ (root mean square). The position of selected points on the right side of the body was recorded by attaching the infrared reflective markers to the skin overlying the following bony landmarks (Fig. 1B): gleno-humeral joint $(\mathrm{GH})$, anterior superior iliac spine (ASIS) and posterior superior iliac spine (PSIS), greater trochanter (GT), lateral femur epicondyle (LE), lateral malleolus (LM), and fifth metatarso-phalangeal joint (VM). ASIS and PSIS coordinates were averaged to obtain ileum (IL) position. Electromyographic (EMG) activity was recorded by means of surface electrodes from the rectus femoris (RF), biceps femoris (long head, BF), lateral gastrocnemius (GCL), and tibialis anterior (TA) using optic fiber BTS TELEMG. EMG signals were preamplified $(\times 100)$ at the recording site, digitized, and transmitted to the remote amplifier via $15-\mathrm{m}$ optic fibers (Grasso et al. 2000). These signals were high-pass filtered (artifact rejection was obtained by cascading 3 1st-order high-pass at $1 \mathrm{~Hz}$ followed by 1 1st-order high-pass at $10 \mathrm{~Hz}$ ), low-pass filtered (200$\mathrm{Hz}$, 4-pole Bessel low-pass), and sampled at $500 \mathrm{~Hz}$. Sampling of kinematic and EMG data were synchronized.

The instantaneous velocity of the treadmill was recorded via an optical encoder (resolution $0.005 \mathrm{~m} \mathrm{~s}^{-1}$ ) and controlled by a computer at a frequency of $30 \mathrm{~Hz}$. We used two different treadmill control modes: 1) constant velocity and 2) variable position-related velocity. In the latter mode the velocity of the treadmill was controlled by a computer using a feedback from the subject's position in such a way that forward displacements from the initial position increased the treadmill velocity while backward displacements decreased it proportionally (Fig. 1, A, C, and D). To measure subject's position, a light-weight stiff thread was attached to the subject (at the level of the waist). The thread was kept in tension by a constant force (about $2 \mathrm{~N}$ )
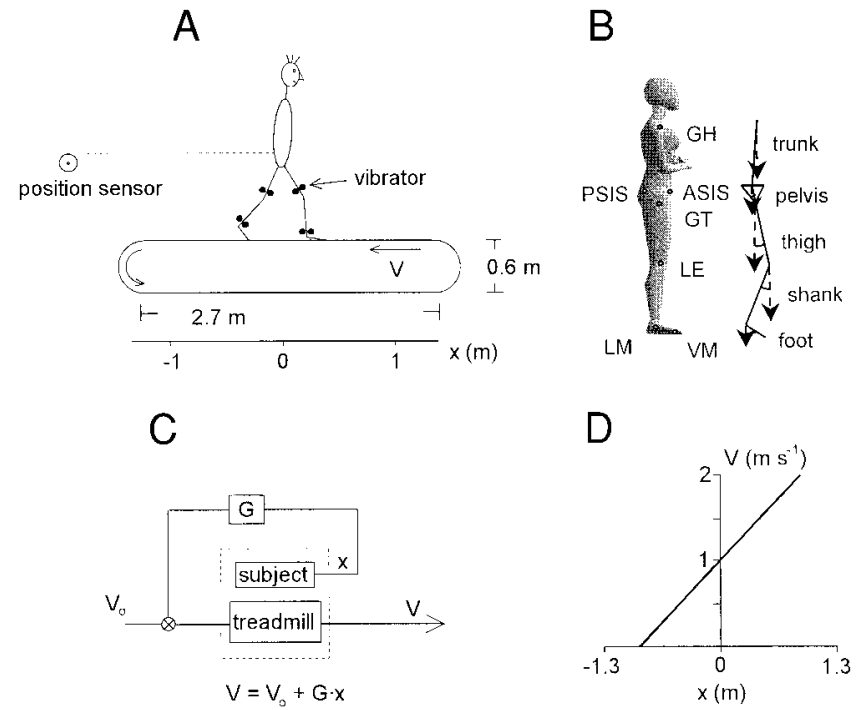

FIG. 1. Experimental setup. A: schematic view of the treadmill and position of vibrators. $B$ : schematic illustration of the body geometry. Markers placed on the right side of the subject were monitored by the ELITE system. From top to bottom: gleno-humeral joint (GH), anterior superior iliac spine (ASIS) and posterior superior iliac spine (PSIS), greater trochanter (GT), lateral femur epicondyle (LE), lateral malleolus (LM), and 5th metatarso-phalangeal joint (VM). ASIS and PSIS coordinates were averaged to obtain ilium (IL) position. Trunk, pelvis thigh, shank, and foot are the limb segments identified by these markers. Elevation angles are computed relative to the vertical (dashed line). $C$ : block diagram of the position-related velocity controller. The subject started to walk from the central position. $D$ : the law of the controller: the treadmill velocity changed proportional to the horizontal displacement of the subject relative to the starting position $(0)$ as measured by a position sensor: a $1-m$ forward body displacement produced a $1.1-\mathrm{m} \mathrm{s}^{-1}$ increase of speed $(G=1.1)$.

produced by a torque-motor (type JR24M4CH, ServoDisc, PMI, Commack, NY). A linear potentiometer on the motor shaft measured the changes of the subject's position (that occurred when the velocity of the subject differed from that of the treadmill belt). Position was sampled at $30 \mathrm{~Hz}$ with an accuracy of $2 \mathrm{~mm}$. The initial subject's position was set in the middle of the treadmill belt. To avoid jerks, the treadmill velocity was low-pass filtered: the transition from one velocity to another was automatically programmed with a ramp profile of $1 \mathrm{~m} \mathrm{~s}^{-2}$ acceleration. This allowed dampening of fast changes in treadmill velocity due to the natural horizontal oscillations of the body (of about $\pm 5 \mathrm{~cm}$ ) (see Thorstensson et al. 1984) during locomotion. The servo provided an efficient and stable control of the treadmill speed in the frequency range of $0-2 \mathrm{~Hz}$. Safety circuits were incorporated in the system. In addition, either the experimental subject or the experimenter could stop the treadmill at any time. The feedback constant $(G=1.1$; see Fig. 1$)$ and treadmill acceleration $\left(1 \mathrm{~m} \mathrm{~s}^{-2}\right)$ were selected to make subjects feel comfortable when they changed their walking speed. This was verified in preliminary experiments. Using such values, the maximum attainable speed increment was $1.3 \mathrm{~m} \mathrm{~s}^{-1}$, and no instability in the feedback control occurred. If so required, the subjects were able to safely increase their walking speed by about $1.3 \mathrm{~m} \mathrm{~s}^{-1}$, up to a maximum walking speed of $2.3 \mathrm{~m} \mathrm{~s}^{-1}$. However, no such high speed increments were observed following the application of mechanical vibration.

\section{Subjects}

It is known that some subjects do not show behavioral effects on muscle vibration (Eklund and Hagbarth 1966; Gurfinkel et al. 1998). We performed experiments on seven normal subjects (age 22-39 yr old, 4 males and 3 females) who participated in earlier experiments with neck muscle vibration (Ivanenko et al. 1999a) and who were sensitive to vibration from the very first application. None of the 
subjects had any history of neurological disease or vestibular impairment. Informed consent was obtained from all participants after the experimental procedure had been explained according to the protocol of the Ethics Committee of the Santa Lucia Institute.

\section{Parameters of vibration}

Vibrators were fastened over a muscle belly or tendon. The following muscles were tested for the effect of vibration: quadriceps (Q), hamstring (HS), triceps surae (TS), and tibialis anterior (TA).

Stimulation of leg muscle proprioceptors $(1.5 \mathrm{~mm}$ amplitude, $80 \mathrm{~Hz}$ sinusoid) was performed using custom-designed electromechanical vibrators (DC motor, equipped with small eccentric rotating masses, weighted about $350 \mathrm{~g}, 9 \mathrm{~cm}$ long with a diameter of $4.5 \mathrm{~cm}$ ) fixed by an elastic belt. In the case of TS muscles, vibrators were fixed to the Achilles tendons, at the level of the ankle joint. In the case of TA muscles, vibrators were fixed on the tendons of TA, $3-5 \mathrm{~cm}$ above the ankle joint. In the case of Q and HS muscles, vibrators were fastened over the muscle belly approximately $15-20 \mathrm{~cm}$ above the knee joint. In all cases, two identical vibrators were synchronized to simultaneously stimulate both legs.

We took particular care to fixate the mechanical vibrators with tight elastic bands so that their motion at the heel impact was minimized. The tension developed by the elastic band ( $3 \mathrm{~cm}$ wide) when wrapped around the thigh was about $7 \mathrm{~N} \mathrm{~cm}^{-1}$, which corresponded to a pressure of about $1 \mathrm{~N} \mathrm{~cm}^{-2}$ (i.e., $75 \mathrm{mmHg}$ acting on the arterial vessel walls, which is about $50 \%$ of the compression value necessary to occlude them while in standing position). To check whether the elastic band affected the blood supply to the leg, one subject underwent routine Doppler ultrasound measurement (Multidop, Esaote, Italy) of blood velocity in the posterior tibialis artery. No change following the fixation of the vibrator on the thigh was detected in standing position. Moreover, during the experiments the vibrators were never kept for more than $5 \mathrm{~min}$, which is not long enough to cause ischemic problems.

In two subjects we checked whether the frequency and amplitude of the vibrator was unstable during the walking cycle. We attached an infrared emitting marker on the right aspect of the vibrator on the right leg and used the OPTOTRAK (Northern Digital, Waterloo, Ontario) system (operating at $1,000 \mathrm{~Hz}$ with a resolution better than $0.1 \mathrm{~mm}$ ) to measure the vibration parameters during locomotion: we found that the frequency and AM along the cycle never exceeded $10 \%$ for all vibrated muscles (Fig. 2A). The switch on/off time for $80-\mathrm{Hz}$ vibration was about $400 \mathrm{~ms}$ with an exponential time course of the frequency change (Fig. 2B): about $200 \mathrm{~ms}$ was needed to reach the $50-\mathrm{Hz}$ frequency.

We evaluated the amount of vibration spreading to the antagonist muscle groups via the tight elastic band by putting an infrared marker on relevant portions of the band. During muscle vibration, oscillations of the marker placed on the band portion overlying the antagonist muscles had an amplitude of $0.1-0.2 \mathrm{~mm}$ (about $10 \%$ of that produced by the vibrator) orthogonal to the skin. Oscillations spred also in the direction parallel to the elastic bend $(0.1-0.5 \mathrm{~mm})$, but their efficiency in stimulating muscle receptors might be limited by the slip of the skin over subcutaneous tissue layers.

\section{Protocol}

Experiments were performed by the subjects with eyes open in dim ambient illumination (since they did not feel comfortable with eyes closed for more than several steps during walking on the treadmill). To record the markers located on the right side of the body, the subjects were asked to keep the arms folded on the chest. In all experiments, subjects were instructed not to resist the applied perturbation.

In the first set of experiments, continuous muscle vibration was tested in the following four conditions.
A

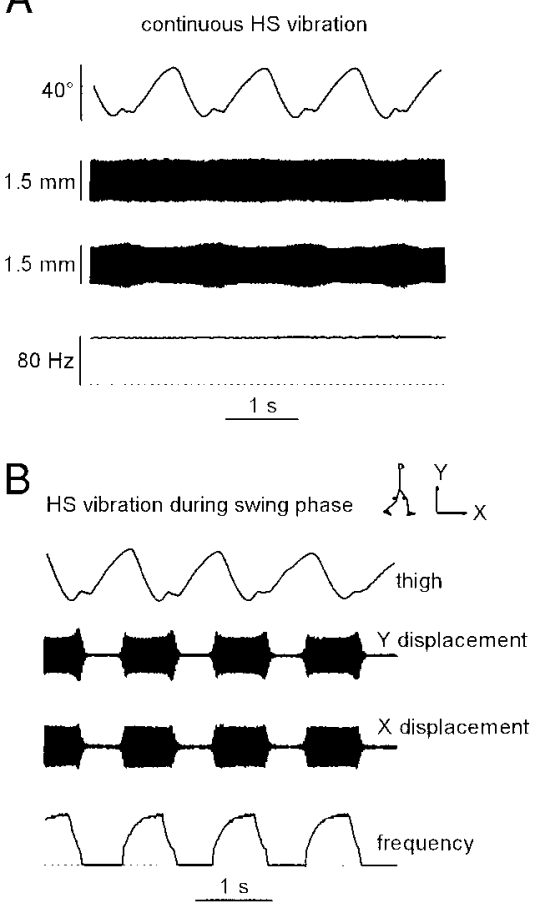

FIG. 2. Instantaneous amplitude and frequency of a vibrator during continuous $(A)$ and phasic (swing phase; $B$ ) hamstring (HS) vibration. For this purpose we attached an infrared emitting marker on the vibrator and used the OPTOTRAK system to measure the vibration parameters during locomotion. The signal was high-pass filtered (15-Hz cutoff). Note a small frequency and $\mathrm{AM}$ along the cycle. For phasic stimulation, the switch on/off time for an $80-\mathrm{Hz}$ vibration was about $400 \mathrm{~ms}$ with an exponential time course of the frequency change: about $200 \mathrm{~ms}$ were needed to reach $50 \mathrm{~Hz}$.

POSTURE. Subjects stood on a force platform (KISTLER 9281B) which we used to measure the displacement of the center of pressure in the sagittal and frontal directions. The centers of the heels were placed on marks $12 \mathrm{~cm}$ apart and the feet splayed out at approximately $30^{\circ}$. After $5 \mathrm{~s}$ of quiet standing, we applied a 6- to 8-s period of muscle vibration.

STEPPING IN PLACE. Subjects were asked to step in place. After about 5-7 s of stepping movements at a comfortable cadence (which occurred in the range $0.5-1 \mathrm{~Hz}$ ), we delivered muscle vibration (for $7-10 \mathrm{~s})$. The speed of the body displacement evoked by muscle vibration was measured by computing the slope of the regression line fitting the displacement of ilium.

TREADMILL LOCOMOTION AT CONSTANT BELT SPEED. After 5-7 s of "steady-state" $1 \mathrm{~m} \mathrm{~s}^{-1}$ locomotion on the treadmill, muscle vibration was applied. Changes of the walking speed on stimulation were allowed within the limits of the treadmill length. Starting position of the subject on the treadmill could be changed so that to use all length of the treadmill. The stimulation was switched off when the subject approached the end of the treadmill belt. Changes in walking speed evoked by muscle vibration gave rise to the subject's displacement on the treadmill (relative to space) and were measured by computing the slope of the regression line fitting the displacement of ilium versus time.

TREADMILL LOCOMOTION AT VARIABLE POSITION-CONTROLLED BELT SPEED. To estimate the time course of speed changes, we set up a walking condition where the subjects were free to change their current speed on the presence of vibration without the risk of reaching the belt bounds. We performed the experiment in the following way: we took the subject to the center of the treadmill belt, then we set the initial speed at $1 \mathrm{~m} \mathrm{~s}^{-1}$ (in some experiments the initial speed was set at $0.5-1.5 \mathrm{~m} \mathrm{~s}^{-1}$ in $0.25 \mathrm{~m} \mathrm{~s}^{-1}$ increments) and let the subject start 
walking (Fig. 1A); then the treadmill velocity was controlled proportional (by $1.1 \mathrm{~m} \mathrm{~s}^{-1}$ per $1 \mathrm{~m}$ displacement) to the changes of subject's position relative to the starting point (Fig. 1D).

The change of walking speed induced by 10 - to 15 -s muscle vibration was calculated as the difference between the mean treadmill velocity (as derived from the optical encoder) during the last $5 \mathrm{~s}$ of muscle vibration and the mean treadmill velocity during $5 \mathrm{~s}$ before vibration.

\section{Perceived body configuration during stepping in place}

In another experiment, we estimated the subjective perception of body configuration during stepping in place movements with and without vibration. We asked the subjects to hold a stable handle with their left hand and to use their right hand to point (eyes closed) with a $25-\mathrm{cm}$ long stick toward the perceived location of the center of their right foot during the stance phase of stepping in place. The position of two infrared active markers attached to the stick was measured by means of a three-dimensional OPTOTRAK system. The line of the stick was extrapolated to where it intersected the ground. This subjective foot placement was compared with the real position of the foot (a 3rd marker was located on the lateral malleolus) with and without vibration.

\section{Phasic vibratory stimulation}

In addition to the standard protocol with continuous muscle vibration, we studied the effect of phasic muscle vibration applied either in swing or stance phase during stepping in place movements and during walking. Walking was performed at about $0.7 \mathrm{~m} \mathrm{~s}^{-1}$ (position-related mode of treadmill velocity control). Two small mechanical footswitches $(6 \times 6 \mathrm{~mm}$, the load to switch them on was $3 \mathrm{~N})$ were located in the subject's shoes at the center of the heel to identify the heel impact (beginning of the stance phase); when the heel was loaded, the switch was on. Phasic muscle vibration was delivered in the current step cycle according to the duration of the previous step cycle. The signal from left and right footswitches was monitored with a sampling rate of $200 \mathrm{~s}^{-1}$, and the current step cycle duration was determined on-line as the time between two successive heel impacts.

The duration of effective vibration corresponded always to $40 \%$ of the previous step cycle duration to avoid phase overlap. To overcome the delay of about $200 \mathrm{~ms}$ needed for the vibrator to reach $50 \mathrm{~Hz}$ (see Fig. $2 B$ ), we anticipated the beginning of the stimulation by $10 \%$ relative to the time of footswitch loading. Thus to trigger muscle vibration in a specific phase of the step cycle, an appropriate delay after the heel impact was programmed: 40 or $90 \%$ of the step cycle to switch on vibration in swing or stance phase, respectively. With a $40 \%$ delay, we obtained a good synchronization with the swing phase and with an additional $50 \%$ shift with the stance phase. The procedure worked because the duration of the ongoing step cycle never changed by more than $10 \%$ of the previous cycle. The left footswitch triggered the vibrator on the left leg, and the right footswitch triggered the vibrator on the right leg.

\section{Data analysis}

The body was modeled as an interconnected chain of rigid segments: GH-IL for the trunk, IL-GT for the pelvis, GT-LE for the thigh, LE-LM for the shank, and LM-VM for the foot (Fig. 1B). The elevation angle of each segment in the sagittal plane corresponds to the angle between the projected segment and the vertical. The limb main axis was defined as the segment connecting GT and LM. The gait cycle duration $(T)$ was defined as the time between two successive maxima of the elevation angle of the limb main axis (Borghese et al. 1996). The stride length during walking on the treadmill was estimated as the treadmill speed times $T$ averaged over several step cycles.
EMGs were numerically rectified and low-pass filtered with cutoff at $50 \mathrm{~Hz}$. EMG data were time normalized over the step cycle duration $(0-100 \%)$ and then averaged over several steps. The intersegmental coordination was evaluated in position space as previously described (Borghese et al. 1996). Briefly, the changes of the elevation angles of the thigh, shank, and foot covary linearly throughout the gait cycle. When thigh, shank, and foot angles are plotted one versus the others in a three-dimensional graph, they describe paths that can be fitted by a plane computed by means of orthogonal linear regression. The orientation of the plane relative to the three axes is measured as the direction cosines of the normal to the plane (i.e., the 3rd eigenvector of the covariance matrix) and quantifies the temporal coupling among the three limb segments (Bianchi et al. 1998).

The change of trunk, shank, and thigh orientation and center-ofpressure displacement induced by 6- to 8-s muscle vibration in quiet standing was calculated as the difference between segment orientation during the last $5 \mathrm{~s}$ of muscle vibration and that during the 5-s period preceding vibration. Statistical analysis (paired $t$-test, within-subjects ANOVA) was performed on the changes in trunk inclination and on the increment of the walking speed evoked by muscle vibration. $P<$ 0.05 was considered significant.

\section{RES ULTS}

\section{Posture}

Muscle vibration during quiet standing evoked typical body responses (Eklund 1972; Lackner and Levine 1979), which are described in Fig. 3. Vibration of TA evoked a prominent forward body tilt, vibration of Achilles tendons induced backward body tilt, HS vibration elicited backward inclination of the trunk and forward inclination of the shank due to hip extension and knee flexion, and vibration of $\mathrm{Q}$ evoked no prominent postural response (Fig. 3). Vibration of thigh muscles did not change significantly the position of the center of the body mass, whereas shank muscle vibration elicited its prominent forward (TA) or backward (TS) displacement.

\section{Stepping in place}

When vibrating hamstring muscles during stepping in place, subjects started moving forward (Fig. 4). From a quantitative viewpoint, the effect of vibration of most muscles during stepping in place differed significantly from that obtained during quiet standing. In standing, vibration of TA and TS

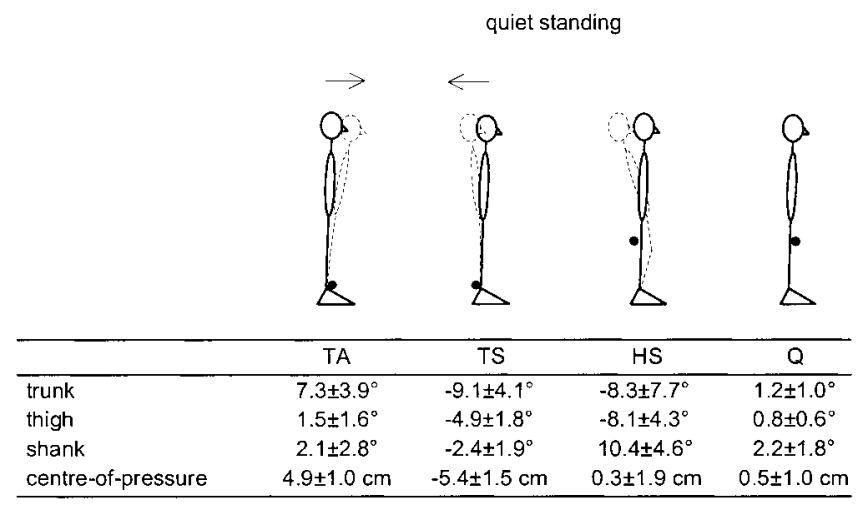

FIG. 3. Changes in trunk, thigh, and shank inclination and in center-ofpressure in the sagittal direction $(n=7$, mean \pm SD) evoked by muscle vibration during quiet standing. Positive values denote forward tilt. A schematic change of the mean body configuration is illustrated in the top panel. TA, tibialis anterior; TS, triceps surae; Q, quadriceps. 
elicited prominent body tilt in all subjects; during stepping, vibration of these muscles evoked only small and inconsistent body displacements. Vibration of Q had little effect both on standing posture and on body displacement during stepping. On the contrary, vibration of HS muscles, which evoked knee flexion and backward trunk inclinations during standing, evoked prominent forward translation in all subjects during stepping in place. Figure $7 \mathrm{~A}$ shows the mean speed of the evoked body displacement induced by the vibration of the four muscle groups.

The mean speed of the evoked body displacement after HS muscle vibration was $0.27 \pm 0.16(\mathrm{SD}) \mathrm{m} \mathrm{s}^{-1}$, while in the control (unperturbed) condition the average speed of the spontaneous subject's displacement during $10 \mathrm{~s}$ of stepping in place was $0.02 \pm 0.02 \mathrm{~m} \mathrm{~s}^{-1}$. We found no evidence for the appearance of tonic muscle activity ${ }^{1}$ in either BF or other muscles following vibration (see Fig. 4). Forward displacement was characterized by the changes of the phase relation among adjacent lower limb segments. This can be appreciated in the three-dimensional (3-D) position space (Fig. 4, bottom). Before vibration, the loops representing intersegmental coordination were close to a straight line due to the fact that the phase shift between adjacent segments was either 0 or $180^{\circ}$. Application of muscle vibration did not increase the stepping frequency (stepping frequency was $0.79 \pm 0.08 \mathrm{~s}^{-1}$ and $0.80 \pm 0.10 \mathrm{~s}^{-1}$ before and during HS vibration, respectively). Instead, it gave rise to a phase shift between thigh and shank segments and a forward translation of the center of body mass. The thighshank-foot 3-D loops during forward translation describe paths that can be fitted by a planar surface $(99 \%$ of variance was explained by the planar regression) (see Borghese et al. 1996). However, motion did not result from a transition to a natural locomotor pattern, as reflected by the differences in the patterns of coordination described by the 3-D gait loops of Figs. 4 and 5. Instead, subjects continued to step in place, but the swinging foot systematically landed in front of the standing foot.

During stepping in place, vibration induced smaller trunk inclinations than in quiet standing, except for the case of $\mathrm{Q}$ vibration whose effect was equally small both during standing or stepping (Table 1).

\section{Walking on the treadmill with a constant belt speed $\left(1 \mathrm{~m} \mathrm{~s}^{-1}\right)$}

In this protocol, the subjects underwent vibration of lower limb muscles while they were walking on the treadmill at $1 \mathrm{~m}$

\footnotetext{
${ }^{1}$ To evaluate the amount of tonic muscle activity induced by HS vibration on BF, we proceeded as follows. Rectified, filtered EMGs were time normalized over the step cycle duration, and then they were ensemble averaged over 4 steps before and during HS vibration. We calculated the mean EMG level in each of 20 consecutive 5\%-wide portions of the step cycle. The lowest value was taken as an estimate of tonic baseline components in the EMG profile. Values were always very low both before $(1.29 \pm 0.23 \mu \mathrm{V}$, about $2 \%$ of peak $\mathrm{BF}$ activity) and during HS vibration $(1.58 \pm 0.32 \mu \mathrm{V})$, the changes being nonsignificant (paired $t$-test: $P>0.08$ ). The changes in the mean rectified EMG activity of all muscles over the whole step cycle displayed considerable inter-individual variability. Mean BF EMG increased in some subjects (up to $30 \%$, the subject shown in Fig. 10) but decreased in others (down to $-60 \%$ ) such that no systematic changes could be detected. Moreover, the changes in mean EMG activity displayed by a given muscle during HS vibration tended to correlate positively with those induced on the same muscle by a voluntary (no vibration) forward stepping at similar speed (RF: $r=0.74, P=0.06$; BF: $r=0.86, P=0.01$; TA: $r=0.17, P=0.56$; GCL: $r=0.75, P=0.05$ ).
}
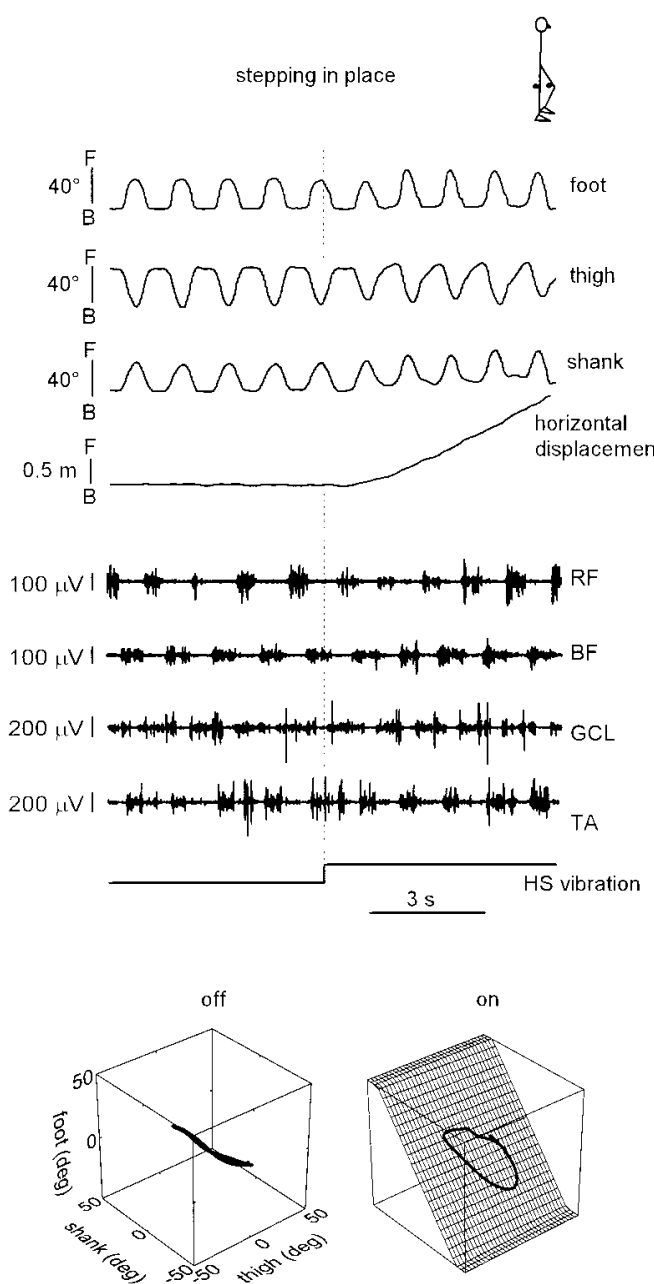

FIG. 4. Kinematics and electromyographic (EMG) activity during stepping in place before and during HS vibration. Bottom plots represent the average thigh-shank-foot loops before (off) and during (on) vibration. During stepping in place without vibration, the 3 elevation angles covary linearly due to in- and opposite-phase changes in the waveforms. During vibration, the elevation angles form a loop that evolves over a plane. F, forward inclination (corresponds to the clockwise rotation of segments on the top schematic drawings); $\mathrm{B}$, backward inclination; RF, rectus femoris; BF, biceps femoris; GCL, lateral gastrocnemius; TA, tibialis anterior.

$\mathrm{s}^{-1}$. Changes of walking speed on stimulation were allowed within the limits of the treadmill length. Displacements tended to develop linearly with time; therefore we measured the increment in walking speed as the slope of the regression line fitting the displacement of the pelvis point versus time during the stimulus. The stimulation was switched off when the subject approached the end of the treadmill belt.

Among the four groups of muscles we studied, the largest increase in speed was found during vibration of HS muscles (by about $25 \%$, Fig. $7 B$ ). Thus the relative effect of muscle vibration was roughly similar to that obtained during stepping in place. The changes in trunk inclination induced by vibration were not significantly different from those induced during stepping in place (Table 1).

\section{Walking with position-related treadmill velocity}

While walking on the treadmill with position-related belt velocity (that is when treadmill velocity was automatically 

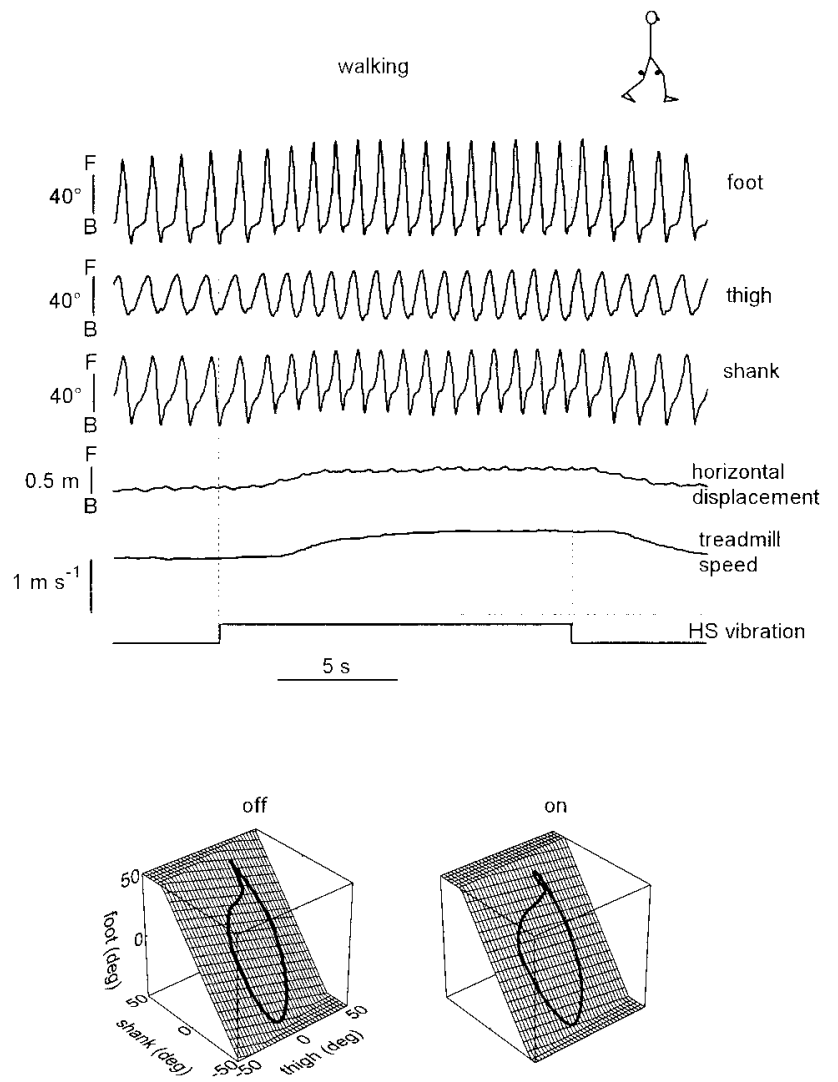

FIG. 5. Changes in kinematics evoked by HS vibration during walking on the treadmill with position-related belt speed (the same subject as on Fig. 4). $\mathrm{F}$, forward inclination (corresponds to the clockwise rotation of segments on the top schematic drawings); B, backward inclination. Bottom plots represent the average thigh-shank-foot loops before (off) and during (on) vibration. Both before and during vibration the loop evolves over a plane.

adjusted to the subject's position), the speed increased often with a ramp-hold profile (Fig. 5) or sometimes progressively during muscle vibration. The relative magnitude of the effect of muscle vibration at the steady-state tended to be slightly larger than that during locomotion with a constant belt velocity (cf. Fig. 7, $B$ and $C$ ). At $1 \mathrm{~m} \mathrm{~s}^{-1}$, HS muscle vibration increased the walking speed by $0.27 \pm 0.17 \mathrm{~m} \mathrm{~s}^{-1}$ for walking with constant treadmill speed, whereas with the position feedback the increase was $0.33 \pm 0.17 \mathrm{~m} \mathrm{~s}^{-1}$. For the other muscles (Q, TA, and TS) the effect of vibration was as small as in the condition without position feedback.

After the cessation of HS vibration, the walking speed returned gradually to the prestimulus level (Fig. 5) or with some overshoot. The walking speed increment induced by HS vibration was accompanied by an increase of the stepping

TABLE 1. Changes in trunk inclination evoked by muscle vibration

\begin{tabular}{|c|c|c|c|c|}
\hline & TA & TS & HS & Q \\
\hline Quiet standing & $7.3 \pm 3.9^{\circ}$ & $-9.1 \pm 4.1^{\circ}$ & $-8.3 \pm 7.7^{\circ}$ & $1.2 \pm 1.0^{\circ}$ \\
\hline Stepping in place & $-1.0 \pm 1.8^{\circ}$ & $-0.4 \pm 0.1^{\circ}$ & $-2.4 \pm 2.2^{\circ}$ & $1.0 \pm 1.1^{\circ}$ \\
\hline $\begin{array}{l}\text { Walking on the } \\
\text { treadmill at } 1 \\
\mathrm{~m} \mathrm{~s}^{-1}\end{array}$ & $0.0 \pm 1.0^{\circ}$ & $0.4 \pm 1.5^{\circ}$ & $0.2 \pm 2.2^{\circ}$ & $1.2 \pm 1.0^{\circ}$ \\
\hline
\end{tabular}

Values are means $\pm \mathrm{SD} ; n=7$. Positive values denote forward tilt of the shoulders. TA, tibialis anterior; TS, triceps surae; HS, hamstring; Q, quadriceps. frequency (paired $t$-test, $P<0.001$ ) and stride length (paired $t$-test, $P=0.14$, not significant; however, 5 of the 7 subjects showed a significant increment). The stepping frequency was $0.82 \pm 0.05 \mathrm{~s}^{-1}$ before the stimulus and $1.03 \pm 0.11 \mathrm{~s}^{-1}$ during the stimulus; the stride length was $1.27 \pm 0.13 \mathrm{~m}$ before the stimulus and $1.32 \pm 0.20 \mathrm{~m}$ during the stimulus.

Figure 6 shows the EMG ensemble average of 15 gait cycles from 1 subject in 2 different steady states: during normal walking at $1.4 \mathrm{~m} \mathrm{~s}^{-1}$ and during walking on the treadmill with position-related belt speed when this speed was achieved by HS muscle vibration. In all examined muscles, the EMG activity was similar as revealed by the high correlation between the plotted waveform pairs (Fig. 6).

The changes in lower limb segment kinematics displayed some amount of interindividual variability. For example, for some subjects (as the one in Fig. 5), there was a prominent change in the amplitude of limb elevation angles during HS vibration. For others, these changes were small. The 3-D gait loops representing intersegmental coordination always evolved over a plane both with and without vibration (99\% of variance was explained by the planar regression). The orientation of this regression plane on vibrating HS muscles also showed some interindividual variability: for example, for four subjects (as the one on Fig. 5), the third eigenvector of the covariance matrix decreased with HS vibration, indicating a change in the phase relation among the three limb segments similar to what happens during natural speed increments (Bianchi et al. 1998);
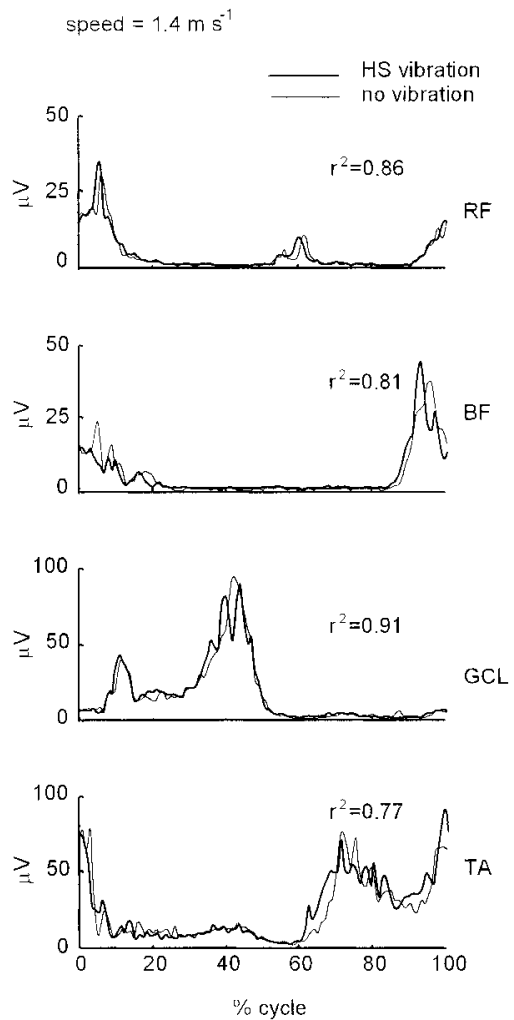

FIG. 6. Ensemble averages of EMG activity from 1 subject during treadmill walking at $1.4 \mathrm{~m} \mathrm{~s}^{-1}$. Thick traces correspond to patterns generated during HS vibration (position-related treadmill control). Thin traces correspond to patterns generated without vibration at the same speed (constant belt velocity). RF, rectus femoris; BF, biceps femoris; GCL, lateral gastrocnemius; TA, tibialis anterior. Insets: squared correlation coefficients between the plotted pairs. 
for three other subjects, it increased. On average, there was no significant change of the third eigenvector $(0.27 \pm 0.10$ before HS vibration and $0.26 \pm 0.12$ during vibration). Thus the increment of the walking speed induced by HS vibration in different subjects was not necessarily accompanied by similar changes in lower limb kinematics and in the orientation of the regression plane as during natural speed increments.

\section{Influence of direction of progression}

The effect of muscle vibration during backward locomotion was tested in the speed range of $0.5-0.75 \mathrm{~m} \mathrm{~s}^{-1}$ (positionrelated mode of treadmill velocity control). Vibrating muscles during backward walking caused different consequences as compared with forward walking (Fig. 7D). Vibration of HS muscles tended to slow locomotion down rather than speeding it up. In contrast, Q vibration, which had little or no effect in forward locomotion, significantly increased the walking speed in the backward direction (paired $t$-test, $P<0.05$ ). For TA and TS muscles, the effect was tiny as in forward locomotion.

\section{Influence of initial velocity}

We tested whether the increment of walking speed evoked by HS vibration depended on the initial "background" velocity. For this purpose we used the position-related mode of treadmill velocity control with different initial velocities (in the range of $0.5-1.5 \mathrm{~m} \mathrm{~s}^{-1}$ ). Figure 8 shows the results of such an experiment. The velocity increment due to muscle vibration tended to decrease slightly with increasing initial walking speed $\left(F_{4,24}=\right.$ 2.97, $P=0.04$, within-subject ANOVA).

It is worth noting that no subject showed a transition from walking to running. In two subjects, we tested the effect of HS muscle vibration at an initial velocity $\left(2 \mathrm{~m} \mathrm{~s}^{-1}\right)$ close to or higher than the spontaneous velocity of transition from walk to run (on average $1.9 \mathrm{~m} \mathrm{~s}^{-1}$ ) (see Thorstensson and Roberthson 1987). Vibration elicited a further increment of the walking
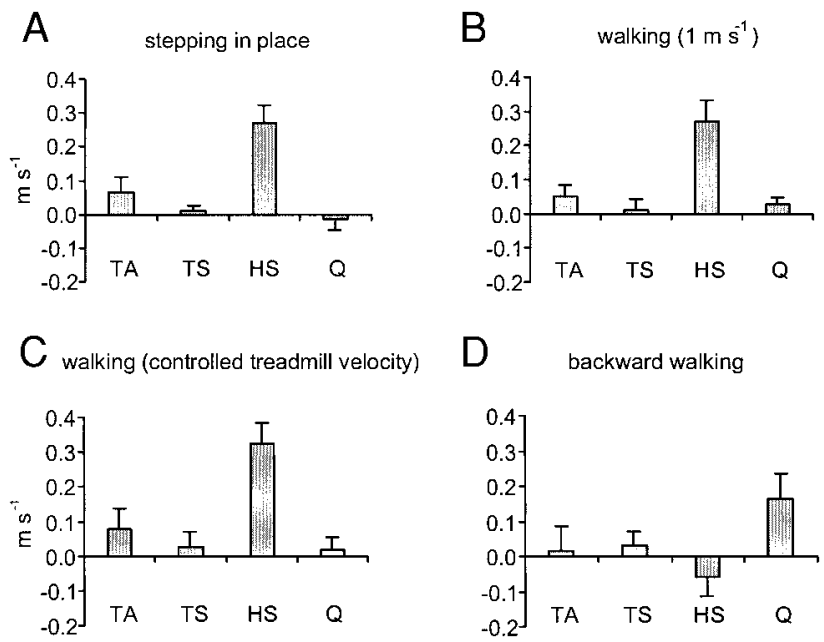

FIG. 7. Speed of forward progression during stepping in place $(A)$ and speed changes during walking $(B-D)$ evoked by continuous muscle vibration. Walking on the treadmill was tested in 3 conditions: with a constant belt velocity $\left(1 \mathrm{~m} \mathrm{~s}^{-1} ; B\right)$ and with position-controlled belt velocity in forward (the initial speed was about $1 \mathrm{~m} \mathrm{~s}^{-1} ; C$ ), and in backward direction (at about $\left.0.5-0.75 \mathrm{~m} \mathrm{~s}^{-1} ; D\right)$. Bars represent mean $\pm \mathrm{SE}$ and were obtained by pooling the data from all subjects $(n=7)$; positive values indicate speed increments regardless of the walking direction.

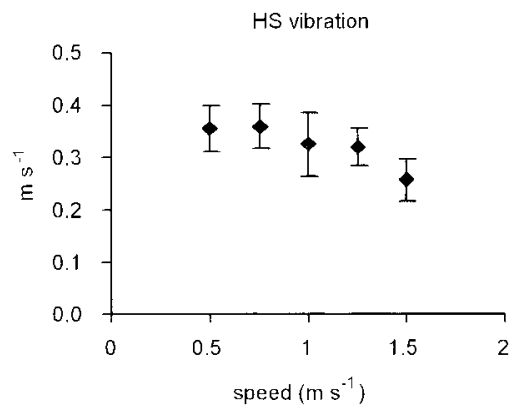

FIG. 8. Walking speed increment (mean $\pm \mathrm{SE}$ ) induced by HS vibration while walking on the treadmill with different initial velocities (from 0.5 to $1.5 \mathrm{~m} \mathrm{~s}^{-1}$ ). The treadmill velocity was controlled according to the subject's position.

speed (by 0.26 and $0.42 \mathrm{~m} \mathrm{~s}^{-1}$ ) but no transition to running. However, the effect of HS vibration was not confined to the walking mode: during running (at $2.3 \mathrm{~m} \mathrm{~s}^{-1}$ ), muscle vibration also elicited a speed increment in the same two subjects (by 0.28 and $0.35 \mathrm{~m} \mathrm{~s}^{-1}$ ).

\section{Continuous versus phasic HS muscle stimulation: vibration during stance and swing phase}

Continuous vibration of HS muscles elicited larger speed increments than phasic stimulation during swing or stance phase both in walking and stepping in place movements (Fig. 9). HS vibration tended to be more effective when applied during swing than during stance phase $\left(F_{1,6}=11.4, P<0.02\right.$, within-subject ANOVA for stepping in place and walking). This effect was most prominent in four subjects and less pronounced in the other three. Nobody showed a larger speed increment during HS vibration in stance phase.

\section{Perceptual effects}

Subjects were aware of the speed increment induced by HS vibration during stepping in place with eyes open or closed as well as during walking with eyes open. All subjects reported that they increased their speed as if "something was pushing me forward." If so required, they could make an effort to resist the imposed perturbation thus suppressing the walking speed increment induced by HS muscle vibration.

We also studied the perceptual effects during stepping in place movements when the subject (with eyes closed) kept a
A

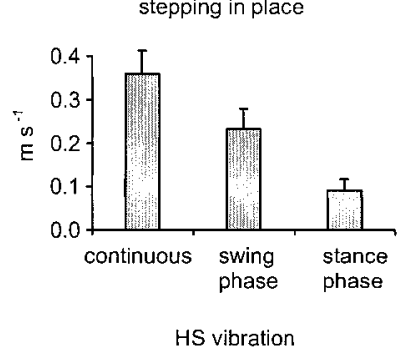

B

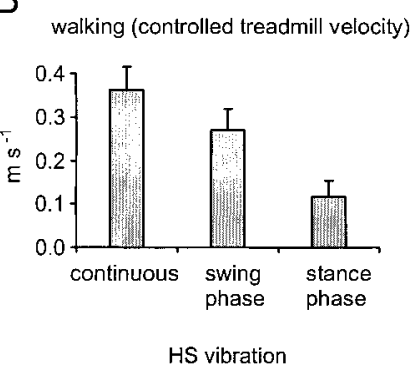

FIG. 9. Speed of forward progression during stepping in place $(A)$ and speed increment during walking on the treadmill with position-controlled belt velocity $(B)$ induced by HS muscle vibration. The initial walking speed was about $0.7 \mathrm{~m} \mathrm{~s}^{-1}$. Three conditions were tested: continuous vibration of HS muscles and HS vibration during swing or stance phase. Bars represent mean $\pm \mathrm{SE}$ 


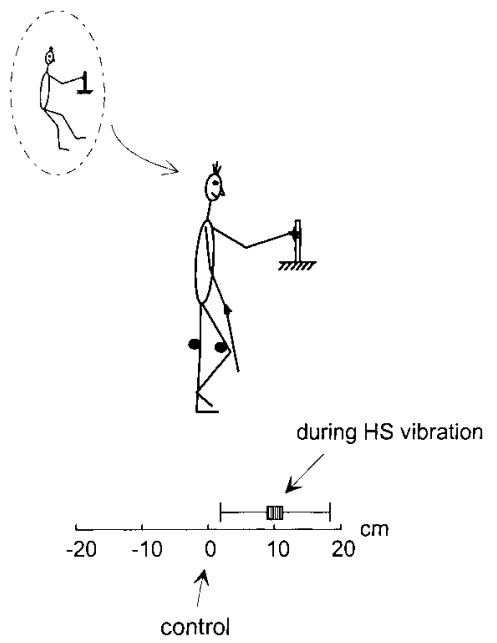

FIG. 10. Illusory change of the foot placement (mean \pm SD) induced by continuous HS muscle vibration during stepping in place movement while the subject (eyes closed) kept a left hand contact with an external immobile object. The subject was asked to point toward the perceived placement of the center of the right foot.

left hand contact with an external still object. In this condition, subjects did not move forward. During HS vibration, however, they perceived an illusory forward leg flexion relative to the trunk (Fig. 10). We characterized this illusion by estimating the subjective foot placement. In the control experiment (eyes closed, no vibration), subjects pointed (with a stick held in their right hand) on average $5 \pm 4 \mathrm{~cm}$ ahead of the right malleolus (where the marker was placed). During HS vibration, they indicated that their feet were displaced forward relative to the control condition (by $10 \pm 8 \mathrm{~cm}$, range 5-30 cm). In contrast, vibration of other muscles did not elicit perceptual changes in the position of the feet relative to the trunk.

\section{I S C U S S I O N}

The results demonstrated that a continuous muscle vibration can make subjects move forward during stepping in place or change their speed of progression during walking. In all conditions, among the muscle groups studied, the most prominent effect was found during vibration of HS muscles.

\section{Task-dependent proprioceptive influences}

The changes in posture observed during muscle vibration (Fig. 3) are in agreement with those reported in previous studies. The posture of the subject is modified during vibration in a manner consistent with the brain interpreting the length of the vibrated muscle as being "longer" than it actually is and compensating for this (e.g., vibration of the TA results in a forward leaning posture). However, it is worth stressing that, in standing posture, muscle vibration induces not local but global reactions related to the change of whole-body orientation relative to the vertical (Eklund 1972; Lackner and Levine 1979; Quoniam et al. 1992; Roll et al. 1989b; Smetanin et al. 1993). These changes are thought to result from a distortion of the internal representation of the postural body scheme evoked by the stimulation of muscle spindles (Gurfinkel 1994; Ivanenko et al. 1999a; Kavounoudias et al. 1999; Lackner and Levine 1979; Lekhel et al. 1997; Massion 1992; Smetanin et al. 1993).
The participation of local reflexes (such as stretch-reflex) is not unequivocal since they are heavily subjected to supraspinal modulation both in standing and locomotion depending on the behavioral and environmental context (Capaday and Stein 1987; Gurfinkel 1994; Ivanenko et al. 1999c; Nashner 1976, 1980).

For locomotion, the relative magnitude and even the direction of the effect of muscle vibration could differ significantly from the effect on quiet posture (Figs. 3 and 7). This rules out the explanation that changes in walking speed due to muscle vibration are merely a mechanical consequence of the postural effects (such as a shift in center of pressure relative to the current support). For example, for shank muscle vibration, there was a small effect on the walking speed in contrast to the noticeable postural changes in normal standing (Figs. 3 and 7). Therefore the role of specific muscles in affecting locomotion cannot be predicted directly from the effect seen in posture.

Also, the effect of vibration of specific muscles for locomotion cannot be entirely predicted from the effect demonstrated during rhythmic leg movements in lying or sitting position. Indeed, it has been found that continuous vibration of leg muscles in humans can produce rhythmic locomotor-like movements of the suspended leg both in forward and backward directions (Gurfinkel et al. 1998, 1999). It was possible to evoke rhythmic movement by vibration of TA, TS, HS, Q, and even muscles that did not participate in "air-stepping" (flexor digitorum brevis). The authors concluded that vibratory-induced afferent input sets into active state the central structures responsible for stepping generation. However, the excitability of central networks, the gating of sensory information, and the role of muscle proprioception might be task-dependent. Actually, upright overground locomotion is different from the cyclic leg movement occurring in the lying or sitting position since a number of physiological variables are controlled in addition to the kinematic variables. For example, the capability to control intentionally the propulsive forces is fundamental for the adaptation of the body's progression, both in speed and direction (Danion et al. 1997). Equilibrium maintenance is required as well for upright locomotion.

Indeed, the increment of the walking speed induced by HS muscle vibration (Fig. 7) may hardly be explained by a nonspecific excitation of the central structures responsible for stepping generation, since 1 ) during backward locomotion, the walking speed tended to decrease after HS vibration (Fig. 7D), 2) during stepping in place movements, HS muscle vibration did not evoke an increase of the stepping frequency, but it evoked a change in the phase shift among lower limb segments and a forward progression (Fig. 4), and 3) the effect of Q, TA, and TS vibration was relatively small (Fig. 7, $A-C$ ) in contrast to that observed in lying position (Gurfinkel et al. 1998). Therefore we suggest that proprioceptive influences are highly task dependent. The differences among vibrated muscles in evoking rhythmic movements in lying position (Gurfinkel et al. 1998) and in affecting stepping and locomotion (Fig. 7) might be related to the mechanisms controlling walking speed and/or equilibrium during upright locomotion.

\section{Locomotor effects of muscle vibration}

The direct inertial forces applied to the moving muscle by the vibrator's movement are unlikely to account for the effect 
seen on vibration of a particular muscle as the subjects wore the vibrators in all the recording phases. On the other hand, although the frequency and the amplitude of the vibrator during the walking cycle were little modulated $(<10 \%$, Fig. $2 B)$, phasic muscle contractions and muscle shortening or lengthening are known to affect the way spindles and tendon organ afferents respond to vibration (Cordo et al. 1993; Prochazka 1996; Roll et al. 1989a). For these reasons, the firing discharge of sensory afferents in leg muscles during the step cycle may have been complex and phase-dependent, rather than tonic. Vibration may excite not only receptors in the muscles under the vibrator head, but also skin receptors and receptors in surrounding deep tissues and joints.

Yet, despite the artificial nature of the proprioceptive stimuli used in the present study, the effect of HS muscle vibration was strong and could thus reflect the importance of the proprioceptive input from these muscles for human locomotion. The influences are in agreement with earlier works on animals: imposed movement around the hip is known to reset and entrain the rhythm during fictive locomotion in spinal and decerebrate cats (Andersson and Grillner 1983). The receptors signaling hip extension are probably the primary and secondary endings of muscle spindles in hip muscles. The contribution of receptors in hip joint is considered to be negligible because anesthetizing hip joint receptors has no influence on the characteristics of entrainment evoked by imposed movements around the hip (Hiebert et al. 1996; Kriellaars et al. 1994; Pearson et al. 1998).

It has been hypothesized that the degree of hip extension is monitored by the CNS to control the instant of transition to the swing phase and thus the timing of the stance phase (Pearson et al. 1998). However, since HS vibration should signal muscle lengthening and, hence, hip flexion, it would be expected to delay, rather than anticipate, the onset of the swing phase and, thus to decrease the walking speed. Furthermore, HS vibration during the swing phase or lift off was more effective than during the stance phase (Fig. 9). It is also worth stressing that, during stepping in place movements, HS muscle vibration did not evoke an increase of the stepping frequency; instead, vibration elicited forward progression (Fig. 4). Therefore it is unlikely that the effects of HS vibration seen in the present experiments can be accounted for by a mechanism that causes an earlier transition from stance to swing phase.

While animal studies have indicated a role for input from muscle proprioceptors in transition from stance to swing, the effects of leg muscle vibration seen in the present studies suggest a broader role of proprioception during human locomotion. For example, muscle proprioception might be highly integrated in a multisensory mechanism controlling body equilibrium during upright locomotion, which is likely different from that during quiet posture. Continuous neck muscle vibration can produce an increment of the walking speed as well (Ivanenko et al. 2000). One can mention the illusory change of leg orientation evoked by HS vibration during stepping in place (Fig. 10) and the absence of this illusion as well as the lack of influences during vibration of Q, TA, and TS muscles. Taking into account the illusion of leg-on-trunk flexion (Fig. 10), one could hypothesize that HS vibration distorts the internal representation of body configuration in such a way that the legs are upright and the trunk is tilted forward, to which the appropriate response would be to step forward or speed up forward gait. Furthermore, natural walking speed increments have been shown to be paralleled by small forward trunk inclinations (Thorstensson et al. 1984). However, the above interpretation is unconvincing for two reasons: 1) the direction of the actual whole-body response is typically opposite to that of the illusory whole-body tilt induced by the stimuli (Fitzpatrick et al. 1994, 1999; Lackner and Levine 1979) and 2) our subjects did never report forward trunk inclination. Instead, they perceived forward foot displacement relative to the subjectively "stable" trunk (Fig. 10).

Whatever the specific mechanism, the present results stress the importance of body scheme mechanisms for the control of walking. This would imply coherence between motor reactions to sensory stimuli and the perceptual interpretation of such stimuli (Calvin-Figuiere et al. 1999; Fitzpatrick et al. 1994, 1999; Gurfinkel 1994; Lackner and Levine 1979). Hamstring muscle is a bi-articular muscle crossing the hip and knee joints. Thus a vibration-induced stimulation is compatible with either the knees being extended or the hips being flexed. Possibly, simultaneous activation of skin receptors evoked by muscle vibration might provide kinesthetic information about joint angles consistent with lengthening of HS muscle (Edin and Johansson 1995). As a result, the internal representation of the position of the center of body mass would be displaced behind the supporting foot (Fig. 10, inset). To restore dynamic equilibrium in the context of this distorted representation, compensatory moments of force would be generated resulting in a forward acceleration of the center of body mass and in the perception of "something pushing forward."

The differential effect of HS vibration in the swing and stance phase (Fig. 9) and the symmetrical influences of HS and $\mathrm{Q}$ vibration during forward and backward locomotion (Fig. 7) could also be related to the mechanism of how the leg movement is represented. Vibration of the lengthening antagonist muscle is known to be critical for accurate perception of movement and limb position (Bullen and Brunt 1986; CalvinFiguiere et al. 1999; Capaday and Cooke 1981; Inglis et al. 1991). For example, for stepping in place movements, hip flexors might act as a prime motor for lifting the leg, and HS muscle might be considered as the antagonist lengthening muscle. Thus HS vibration could be expected to evoke larger influences. The modification of motor synergies from forward to backward locomotion (Grasso et al. 1998) might also account for the fact that the proprioceptive stimulation of thigh muscles alters the walking speed depending on the direction of progression (Fig. 7).

A fundamental feature of locomotion is the continuous displacement of the center of body mass relative to the foot contact with the support surface. It is possible that the velocity and the position of the foot relative to the trunk plays a key role in sensing and controlling body movement and foot placement. Even at the level of the spinal cord, sensory neurons in the dorsal spinocerebellar tract encode foot location relative to the trunk (whole-limb orientation) rather than localized proprioceptive information (Bosco et al. 1996). For geometrical reasons, foot location is more sensitive to the changes in proximal rather than distal angles, and this factor may account for the lack of significant influences from shank muscle vibration. The velocity of lower limb excursion relative to the trunk may be derived from hip muscle proprioceptors. How the increase of the total spindle activity evoked by HS muscle vibration is 
interpreted and utilized by the brain networks controlling locomotion is unknown. The regulation of the interactions between posture and locomotion might be critical: the increment in walking speed could represent a dynamic adjustment caused by a distorted representation of body motion in space.

As a general conclusion, the findings highlight the importance of the proprioceptive input from muscle spindles for maintaining the steady state of human locomotion. The results demonstrated that the proprioceptive influences are strongly dependent on the task (for example, normal standing vs. locomotion vs. rhythmic leg movements in lying position) and highly specific. We suggest that proprioceptive information about the movement of the foot relative to the trunk is important for the control of the walking speed in normal locomotion.

The authors thank B. Bronzi and D. Prissinotti for skillful technical help and Dr. E. Troisi for color Doppler sonography measurements.

This work was supported in part by grants from the Italian Health Ministry, the Italian Space Agency, the Ministero della Universita e Ricerca Scientifica e Tecnologica, and Telethon-Italy.

\section{REFERENCES}

ANDERSSON O AND GRILLner S. Peripheral control of the cat's step cycle. II. Entrainment of the central pattern generators for locomotion by sinusoidal hip movements during "fictive locomotion." Acta Physiol Scand 118: 229 239, 1983.

ARMSTRONG DM. Review lecture: the supraspinal control of mammalian locomotion. J Physiol (Lond) 405: 1-37, 1988.

Bianchi L, ANGelini D, ORANi GP, AND LacQuaniti F. Kinematic coordination in human gait: relation to mechanical energy cost. J Neurophysiol 79: 2155-2170, 1998.

Bianconi R AND VAN DER MEUlen JR. The response to vibration of the end organ of mammalian muscle spindles. J Neurophysiol 26: 177-190, 1963.

BorgheSe NA, BIANCHI L, AND LACQUANITI F. Kinematic determinants of human locomotion. J Physiol (Lond) 494: 863-879, 1996.

Bosco G, Rankin A, and Poppele R. Representation of passive hindlimb postures in cat spinocerebellar activity. J Neurophysiol 76: 715-726, 1996.

BULLEN AR AND BRUNT D. Effects of tendon vibration on unimanual and bimanual movement accuracy. Exp Neurol 93: 311-309, 1986.

Burke D, Hagbarth KE, Lofstedt L, AND Wallin BG. The responses of human muscle spindle endings to vibration during isometric contraction. J Physiol (Lond) 261: 695-711, 1976.

Calvin-Figuiere S, Romaiguere P, Gilhodes JC, and Roll JP. Antagonist motor responses correlate with kinesthetic illusions induced by tendon vibration. Exp Brain Res 124: 342-350, 1999.

CAPADAY C AND COOKE JD. The effects of muscle vibration on the attainment of intended final position during voluntary human arm movements. Exp Brain Res 42: 228-230, 1981

CAPADAY C AND Stein RB. Difference in the amplitude of the human soleus $\mathrm{H}$ reflex during walking and running. $J$ Physiol (Lond) 392: 513-522, 1987.

Conway BA, Hultborn H, and Kiehn O. Proprioceptive input resets central locomotor rhythm in the spinal cat. Exp Brain Res 68: 643-656, 1987.

Cordo P, Gandevia SC, Hales JP, Burke D, and Laird G. Force and displacement-controlled tendon vibration in humans. Electroencephalogr Clin Neurophysiol 89: 45-53, 1993.

Cordo P, Gurfinkel VS, Bevan L, and Kerr GK. Proprioceptive consequences of tendon vibration during movement. J Neurophysiol 74: 1675$1688,1995$.

Danion F, BonnaRd M, and Pailhous J. Intentional on-line control of propulsive forces in human gait. Exp Brain Res 116: 525-538, 1997.

EDIN BB AND JOHANSSON N. Skin strain patterns provide kinaesthetic information to the human central nervous system. J Physiol (Lond) 487: 243$251,1995$.

EKLUND G. General features of vibration-induced effects on balance. Ups J Med Sci 77: 112-124, 1972.

EKLUND G AND HAGBARTH KE. Normal variability of tonic vibration reflexes in man. Exp Neurol 16: 80-92, 1966.

FITZPATRICK R, BURKE D, AND GANDEVIA SC. Task-dependent reflex responses and movement illusions evoked by galvanic vestibular stimulation in standing humans. J Physiol (Lond) 478: 363-372, 1994
FITZPATRICK RC, WARDMAN DL, AND TAYLOR JL. Effects of galvanic vestibular stimulation during human walking. J Physiol (Lond) 517: 931-939, 1999.

Forssberg H, Grillner S, Halbertsma J, and Rossignol S. The locomotion of the low spinal cat. II. Interlimb coordination. Acta Physiol Scand 108: 283-295, 1980

GANDEVIA SC. Kinesthesia: roles for afferent signals and motor commands. In: Handbook of Physiology. Exercise. Regulation and Integration of Multiple Systems. Bethesda, MD: Am. Physiol. Soc., 1996, sect. 12, p. 128-172.

Goodwin GM, McCloskey DI, and Matthews PB. The contribution of muscle afferents to kinaesthesia shown by vibration induced illusions of movement and by the effects of paralysing joint afferents. Brain 95: 705$748,1972$.

GRasso R, BIANCHI L, AND LACQUANiti F. Motor patterns for human gait: backward versus forward locomotion. J Neurophysiol 80: 1868-1886, 1998.

GRASSO R, ZAGO M, AND LACQUANITI F. Interactions between posture and locomotion: motor patterns in humans walking with bent posture versus erect posture. J Neurophysiol 83: 288-300, 2000.

Grillner S. Control of locomotion in bipeds, tetrapods, and fish. In: Handbook of Physiology. The Nervous System. Motor Control. Bethesda, MD: Am. Physiol. Soc., 1981, sect. 1, vol. 2, part 1, p. 1179-1236.

GRILlNer S AND Rossignol S. On the initiation of the swing phase of locomotion in chronic spinal cats. Brain Res 146: 269-277, 1978.

GURFINKEL VS. The mechanisms of postural regulation in man. In: Soviet Scientific Reviews, Physiology and General Biology Reviews. Yverdon, Switzerland: Harwood Academic Publishers GmbH, 1994, sect. F, vol. 7, part 5 , p. $59-89$.

GuRfinkel VS, Ivanenko YP, Levik YS, Kazennikov OV, and Selionov VA. The neural control of posture and locomotion: a lock with two keys. In: Motor Control Today and Tomorrow. Sofia, Bulgaria: Academic Publishing House, 1999, p. 113-120.

GuRfinKel VS, LeVIK YS, KaZennikov OV, AND Selionov VA. Locomotorlike movements evoked by leg muscle vibration in humans. Eur J Neurosci 10: $1608-1612,1998$.

Hiebert GW, Whelan PJ, Prochazka A, and Pearson KG. Contribution of hind limb flexor muscle afferents to the timing of phase transitions in the cat step cycle. J Neurophysiol 75: 1126-1137, 1996.

INGLIS JT, FRANK JS, AND INGLIS B. The effect of muscle vibration on human position sense during movements controlled by lengthening muscle contraction. Exp Brain Res 84: 631-634, 1991.

IVANENKO YP, GRASSO R, AND LACQUANITI F. Effect of gaze on postural responses to neck proprioceptive and vestibular stimulation in humans. J Physiol (Lond) 519: 301-314, 1999a.

IVANENKO YP, GRASSO R, AND LACQUANITI F. Walking speed can be modulated by muscle vibration in humans. Soc Neurosci Abstr 25: 2182, 1999b.

IVANENKO YP, GRASSO R, AND LACQUANITI F. Neck muscle vibration makes walking humans accelerate in the direction of gaze. J Physiol (Lond) 525: 803-814, 2000

IVANENKo YP, TALIS VL, AND KaZENniKov OV. Support stability influences postural responses to muscle vibration in humans. Eur J Neurosci 11: $647-654,1999 \mathrm{c}$

Kavounoudias A, Gilhodes JC, Roll R, and Roll JP. From balance regulation to body orientation: two goals for muscle proprioceptive information processing? Exp Brain Res 124: 80-88, 1999.

KriellaARs DJ, Brownstone RM, Noga BR, AND JoRdan LM. Mechanical entrainment of fictive locomotion in the decerebrate cat. J Neurophysiol 71: 2074-2086, 1994.

LACKNER JR AND LEvine MS. Changes in apparent body orientation and sensory localization induced by vibration of postural muscles: vibratory myesthetic illusions. Aviat Space Environ Med 50: 346-354, 1979.

LACQUANITI F. Frames of reference in sensorimotor coordination. In: Handbook of Neuropsychology. Amsterdam: Elsevier Science B.V., 1997, p. $27-64$.

Lacquaniti F, Grasso R, and Zago M. Motor patterns in walking. News Physiol Sci 14: 168-174, 1999.

Lekhel H, Popov K, Anastasopoulos D, Bronstein A, Bhatia K, Marsden $\mathrm{CD}$, AND GRESTY M. Postural responses to vibration of neck muscles in patients with idiopathic torticollis. Brain 120: 583-591, 1997.

MASSION J. Movement, posture and equilibrium: interaction and coordination. Prog Neurobiol 38: 35-56, 1992.

Matthews PBC and Stein RB. The sensitivity of muscle spindle afferents to small sinusoidal changes of length. J Physiol (Lond) 200: 723-743, 1969. 
Mori S, Kawahara K, Sakamoto T, Aoki M, and Tomiyama T. Setting and resetting of level of postural muscle tone in decerebrate cat by stimulation of brain stem. J Neurophysiol 48: 737-748, 1982.

NASHNER LM. Adapting reflexes controlling the human posture. Exp Brain Res 26: 59-72, 1976.

NASHNER LM. Balance adjustments of humans perturbed while walking. J Neurophysiol 44: 650-664, 1980.

Orlovski GN, Deliagina TG, AND GriLlner S. Neural control of locomotion. Form mollusc to man. Oxford, UK: Oxford Univ. Press, 1999.

PEARSON KG, MisiaszeK JE, AND FouAd K. Enhancement and resetting of locomotor activity by muscle afferents. Ann NY Acad Sci 860: 203-215, 1998.

Prochazka A. Proprioceptive feedback and movement regulation. In: Handbook of Physiology. Exercise. Regulation and Integration of Multiple Systems. Bethesda, MD: Am. Physiol. Soc., 1996, sect. 12, p. 89-127.

Quoniam C, Roll JP, DeAT A, And Massion J. Proprioceptive induced interactions between segmental and whole body posture. In: Disorders of Posture and Gait. New York: Georg Thieme Verlag Stuttgart, 1990, p. $194-197$.

Roll JP, VEDEL JP, AND RIBOT E. Alteration of proprioceptive messages induced by tendon vibration in man: a microneurographic study. Exp Brain Res 76: 213-222, 1989a.
Roll JP, Vedel JP, AND Roll R. Eye, head and skeletal muscle spindle feedback in the elaboration of body references. Prog Brain Res 80: 113-123, $1989 \mathrm{~b}$.

Rossignol S. Visuomotor regulation of locomotion. Can J Physiol Pharmacol 74: 418-425, 1996.

SHIK ML AND ORLOVSKY GN. Neurophysiology of locomotor automatism. Physiol Rev 56: 465-501, 1976.

Smetanin BN, Popov KE, and Shlykov VY. Postural responses to vibrostimulation of neck muscle proprioceptors in humans. Neurophysiology (Kiev) 25: 86-92, 1993.

Soechting JF And Flanders M. Moving in three-dimensional space: frames of reference, vectors, and coordinate systems. Annu Rev Neurosci 15: 167-191, 1992.

Thorstensson A, Nilsson J, CARlson H, And Zomlefer MR. Trunk movements in human locomotion. Acta Physiol Scand 121: 9-22, 1984.

THORSTENSSON A AND ROBERTHSON H. Adaptations to changing speed in human locomotion: speed of transition between walking and running. Acta Physiol Scand 131: 211-214, 1987.

Tiemersm D. Body Schema and Body Image: An Interdisciplinary and Philosophical Study. Amsterdam/Lisse: Swets and Zeitlinger B.V., 1989. 